\title{
Super-EBA and IRM for retrograde root filing
}

\author{
Abstracted from
}

Wälivaara DÅ, Abrahamsson P, Fogelin M, Isaksson S.

Super-EBA and IRM as root-end fillings in periapical surgery with ultrasonic preparation: a prospective randomized clinical study of 206 consecutive teeth. Oral Surg Oral Med Oral Pathol Oral Radiol Endod 2011; 112: 258-263. Epub 2011 Mar 31.

Address for correspondence: Dan-Åke Wälivaara, Maxillofacial Unit,

Halmstad Hospital 13, SE-301 85 Halmstad, Sweden. E-mail: dan-ake.valivaara@regionhalland.se

\section{Question: Is there a difference in outcome between retrograde root fillings with IRM and super-EBA?}

\section{Design Randomised controlled trial.}

Intervention Two hundred and six consecutive teeth in 164 patients (99 women and 65 men) were prepared using ultrasonic retrograde root canal treatment and randomly assigned to restoration with either IRM (99 teeth) or super-EBA (107 teeth) using a standard randomisation table. Only those teeth with obvious root fractures or advanced periodontal disease were excluded. Teeth were evaluated at least one year post-operatively (range 12-21 months, average 13) clinically and radiographically. [eg number of participants, inclusion/ exclusion criteria, number receiving intervention, description of intervention, randomisation, blinding, etc.]

Outcome measures Outcomes were success or failure. Clinically failure was apparent if there was tenderness on percussion or palpation of the crown and/or in the apical area, gingival swelling or presence of a fistula or an apicomarginal communication. Radiographically, failure was when there was evidence of uncertain healing or unsatisfactory healing. Complete healing and scar formation were considered to be successful.

Results One hundred and ninety-four teeth ( 96 IRM and 98 SuperEBA) in 153 patients were assessed. The drop-outs were eight teeth (two IRM and six Super-EBA) in seven patients. Four teeth (one IRM and three Super-EBA) were excluded owing to root fractures discovered during the follow-up period. There was no statistical significance between the two groups (IRM and Super-EBA) regarding the healing outcome as it applied to all types of teeth. Of the IRM teeth 87 (90.6\%) were successful. Of the Super-EBA group 80 were successful $(81.6 \%)$ - no confidence intervals are reported but $\mathrm{p}=0.096$ and is therefore not significant.

Conclusions Both IRM and Super-EBA can serve as satisfactory retrograde root-filling materials.

\section{Commentary}

There are many dental restorative materials available which may be used as a retrograde seal during periradicular surgery: amalgam, zinc oxide eugenol based cements and mineral trioxide aggregate to name but a few. The appropriate selection, and the purported evidence for and against these materials, can be confusing. This article aims to compare directly the outcome of periradicular surgery using IRM and Super-EBA retrograde filling materials.

Super-EBA is a general purpose zinc oxide eugenol cement reinforced with ethoxy benzoic acid (EBA) which may be used in crown cementation, temporary dressing or as a cavity liner. It has a neutral $\mathrm{pH}$ and hydrophilic properties, allowing it to be easily utilised in the surgical field. IRM is a zinc oxide eugenol based cement reinforced with polymethacylate. Unlike Super-EBA, it cannot be added to incrementally in the clinical environment.

This study examined 164 consecutive patients referred for apical surgery on all types of teeth. They were randomised into two groups to receive one of the two retrograde sealers, surgery performed and then the patients reviewed at least twelve months later. One hundred and fifty-three patients and 194 teeth completed the trial; $90.6 \%$ of the IRM group and $81.6 \%$ of the Super-EBA group were judged to be a success. The authors speculated this difference may be due to difficulties in the handling characteristics of Super-EBA, resulting in a reduced height of this retrograde seal. The reader should interpret the results of the study with caution. On the face of it, the aims of the article were clearly stated, ethical approval obtained, the surgical procedure well documented and the clinical and radiographic review protocols outlined. In-depth appraisal reveals limited information regarding the population studied and few results regarding the preoperative clinical assessment. Eighty-one per cent of the teeth were judged to have 'uncomplete, shortage or overfilled' orthograde root fillings pre-operatively, but the quality of this obturation was not considered in the inclusion criteria for the study; all teeth referred to the department for apical surgery underwent a surgical procedure. This goes against the advice in the opening paragraph of the article 'Conventional endodontic treatment... if it fails, revision of the orthograde root filling should be considered first'.

\section{Practice point}

- The success of periradicular surgery may be judged both clinically and radiographically. The two parameters should be judged independently of each other both pre- and post-operatively.

Gillian Ainsworth

Department of Oral Surgery, Glasgow Dental Hospital and School, Glasgow, Scotland, UK

Evidence-Based Dentistry (2012) 13, 20. doi:10.1038/sj.ebd.6400843 